\author{
ISOLATION OF CANDIHEXIN- \\ PRODUCING STRAINS OF \\ STREPTOMYCES VIRIDOFLAVUS \\ Lloyd E. McDaniel \\ WAKSMAN Institute of Microbiology, Rutgers, \\ The State University \\ New Brunswick, New Jersey, 08903, U.S.A.
}

(Received for publication November 27, 1975)

Previous reports from this laboratory ${ }^{1 \sim 4)}$ have described the production, isolation, purification, and properties of candihexin, a new hexaene macrolide which is produced by a mutant strain of Streptomyces viridoflavus, IMRU 3961. The parent S. viridoflavus, IMRU 3685, produces candidin, a heptaene. ${ }^{5)}$ Candihexin and candidin are clearly distinguishable by their ultraviolet-visible light spectra $^{2)}$ and by the components which make up the two complexes. ${ }^{4)}$

In this report the procedures are described by which candihexin-producing mutants as well as non-producing mutants have been isolated. The cultural characteristics of the candihexin- and candidin-producing strains are compared.

Isolation of S. viridoflavus IMRU 3961

In a long series of transfers of $S$. viridoflavus IMRU 3685 on $1 \%$ yeast extract$1 \%$ glucose-agar slants, one culture (18A) which was transferred at three-week intervals for a year was found to produce a substance with a hexaene macrolide ultraviolet-visible spectrum..$^{6)}$ The product had maxima (in methanol) at $379,357,340$ and $323 \mathrm{~nm}^{4}$ ) The candidin maxima (in ethanol) are at 406 , 383, 363 and $340 \mathrm{~nm}^{\mathrm{5}}$ ) Isolate 18A2 (now designated IMRU 3961) was obtained by plating a fragmented vegetative culture of $18 \mathrm{~A}$. Only colorless colonies appeared on the plates; there were no yellow colonies typical of the parent strain.

Comparison of mutant IMRU 3961 with parent IMRU 3685

The two cultures were compared by the test procedures used in the International
Streptomyces Project (ISP). ${ }^{7)}$ Culture IMRU 3685 conformed in all respects but one with the characteristics of $S$. viridoflavus as described in the ISP report. ${ }^{8)}$ The exception was that in our hands the culture grew well on the Pridham and Gottlieb medium containing D-glucose. Mutant IMRU 3961 differed from IMRU 3685 only in lacking a yellow color on all solid media used and in growing on $i$-inositol (slight growth after three days, moderate after seven, and heavy after ten).

\section{Plating tests}

In order to find out if candihexin mutants can be isolated directly from IMRU 3685, a shake flask vegetative culture was fragmented in a Waring blendor, centrifuged slowly to remove clumps and large strands, and plated on ISP No. 2 medium. ${ }^{7)}$ The colony types obtained were as follows:

A. Yellow colonies

In three separate platings (in all approximately 4,500 colonies), yellow colonies typical of the candidin producer constituted 97 $98.5 \%$ of the total. Several random yellow isolates were tested and all produced a heptaene. All the yellow colonies were presumed to be candidin-producers.

B. Colorless colonies

One and one-half to three percent of the colonies were without pigment (essentially the same color as the medium). Twelve random isolates were tested in shaken flasks in a medium which supports the production of both candidin and candihexin. No polyene macrolide spectrum was given by the butanol extracts of any of the twelve. Therefore, these colonies (colorless mutants) were classified as non-polyene-producers. Two of these were fragmented and replated. Only colorless colonies were found. Thus, there was no indication of reversion to yellow colonies (candidin-producers).

Productivity tests of colorless colonies were made on solid media. Portions of the colorless colonies were transferred to duplicate plates of ISP No. 2 medium, four isolates per plate plus an IMRU 3961 control. After 4 
days of incubation one set of plates was flooded with a suspension of spores of Aspergillus niger ATCC 10254, drained, and incubated for two days. After incubation the plates were examined for zones of inhibition.

Of 59 colorless colonies tested, eight gave detectable zones of inhibition $(2 \sim 5 \mathrm{~mm}$ from the edges of the colonies) and 51 gave no zones. Each of the eight active cultures was grown in shake flasks and assayed for polyene macrolide production. Of these, one gave a typical heptaene spectrum $(125 \mu \mathrm{g} / \mathrm{ml}$ calculated as candidin), four gave traces of heptaene, two gave no detectable polyene spectrum, and one ( $\$ 87-78)$ gave a typical hexaene spectrum $(220 \mu \mathrm{g} / \mathrm{ml}$ calculated as candihexin).

A concentrated butanol extract of isolate $\# 87-78$ was developed on a thin-layer chromatographic plate and the components were analyzed by spectrodensitometric scan. ${ }^{9)}$ The components were found to be the same as those in candihexin. ${ }^{4)}$ Component $\mathrm{E}$ was present in a somewhat larger amount than is found typically in candihexin but on a single test this difference is not believed to be significant. Tentatively, we consider cultures IMRU 3961 and \$87-78 to be the same and the products of both to be candihexin complex.

Papillae formation

After long incubation of ISP No. 2 medium plates $(14 \sim 21$ days $)$ both the yellow candidin colonies and the colorless non-producer colonies developed distinct papillae. In yellow colonies both yellow and colorless papillae were formed; in colorless colonies only colorless papillae were found. The candihexin culture, IMRU 3961, treated in the same way produced no papillae.

The polyene macrolide production capacities of papillae-derived strains have not been investigated. However, the occurrence of papillae suggests a colony manifestation of the mutations already found to occru in submerged mycelial cultures. Papillae formation may have occurred (and have been unnoticed). in the original 18A slant culture, followed by overgrowth by the candihexin mutant.

\section{References}

1) Martin, J. F. \& L. E. McDaniel: The submerged culture production of the polyene antifungal antibiotics candidin and candihexin. Devel. Ind. Microbiol. 15: 324 337, 1974

2) Martin, J. F. \& L. E. McDaniel: Isolation, purification and properties of the hexaene macrolides candihexin I and candihexin II. J. Antibiotics 27: 610 619, 1974

3) Martin, J. F. \& L. E. McDaniel: Sequence of biosynthesis of the components of the polyene macrolides candidin and candihexin: macrolide aglycones as intracellular components. Antimicr. Agents \& Chemoth. 7: 208 214, 1975

4) Martin, J. F. \& L. E. McDaniel: The candihexin polyene macrolide complex: physicochemical characterization and antifungal activities of the single components. Antimicr. Agents \& Chemoth. 8: 200 208, 1975

5) Taber, W. A.; L. C. Vining \& S. A. WAKSMAN: Candidin, a new antifungal antibiotic produced by Streptomyces viridoflavus. Antibiot. \& Chemoth. 4: 455 461, 1954

6) McDaniel, L.E. \& E. G. Bailey: Liquid nitrogen preservation of standard inoculum: gas-phase storage. Appl. Microbiol. 16: 912 916, 1968

7) Shirling, E. B. \& D. Gottlieb: Methods for characterization of Streptomyces species. Internat. J. Systemat. Bacteriol, 16: 313 340, 1966

8) Shirling, E. B. \& D. Gottlieb: Cooperative description of the type strains of Streptomyces. V. Additional descriptions. Internat. J. Systemat. Bacteriol. 22: 265 394, 1972

9) Martin, J. F. \& L. E. McDaniel: Quantitative thin-layer spectro-densitometric determination of the components of polyene macrolide antibiotic complexes. J. Chromatogr. 104: $151 \sim 160,1975$ 